\title{
Erratum: \\ Critical phenomena in one dimension from a Bethe ansatz perspective
}

\author{
Xiwen Guan \\ State Key Laboratory of Magnetic Resonance and Atomic and Molecular Physics, \\ Wuhan Institute of Physics and Mathematics, Chinese Academy of Sciences, \\ Wuhan 430071, P. R. China \\ Department of Theoretical Physics, Research School of Physics and Engineering, \\ Australian National University, Canberra ACT 0200, Australia \\ xiwen.guan@anu.edu.au
}

Published 10 September 2014

In the paper Int. J. Mod. Phys. B 28, 1430015 (2014), the equation (9) should be replaced by

$$
v_{s} \approx \frac{\hbar \pi n}{m}\left(1-\frac{4}{\gamma}+\frac{12}{\gamma^{2}}+\frac{16}{\gamma^{3}}\left(\frac{\pi^{2}}{3}-2\right)\right)
$$

In addition, here we would like to mention that the exact results for various observables of the Lieb-Liniger model at $T=0$ and at finite temperature were obtained by using field theory methods. The repulsive Lieb-Liniger Bose gas can be obtained as the nonrelativistic limit of the sinh-Gordon model. ${ }^{1,2}$ The local twoand three-body correlations have been calculated from the powerful field theory methods. ${ }^{3,4}$ On the other hand, large spin Bose gases have a rich phase diagram and exhibit various phases of quantum liquids. ${ }^{10,11} \mathrm{~A}$ pedagogical review of theoretical results for the Ising model universality class, including $E_{8}$ S-matrix, correlation functions, integrability breaking, etc, was discussed in terms of integrable field theory and critical phenomena. ${ }^{12}$

Moreover, the generalized Gibbs ensemble ${ }^{5-9}$ is used in discussions of nonthermal distributions in isolated systems with conserved laws. Recently there have been several papers focusing on the study of quench dynamics in regard to the validity of the generalized Gibbs Ensemble (GGE), see Refs. 13-17, etc. This research 


\section{Guan}

has been becoming a new frontier in cold atoms and condensed matter physics. However, understanding thermalization of isolated many-body systems still imposes a big theoretical challenge.

\section{References}

1. M. Kormos, G. Mussardo and A. Trombettoni, Phys. Rev. A 81, 043606 (2010).

2. M. Kormos, G. Mussardo and B. Pozsgay, JSAT/2010/P05014.

3. M. Kormos, G. Mussardo and A. Trombettoni, Phys. Rev. Lett. 103, 210404 (2009).

4. M. Kormos, G. Mussardo and A. Trombettoni, Phys. Rev. A 83, 013617 (2011).

5. M. Rigol, V. Dunjko and M. Olshanii, Nature 452, 854 (2008).

6. J.-S. Caux and F. H. L. Essler, Phys. Rev. Lett. 110, 257203 (2013).

7. J. Nardis, B. Wouters, M. Brockmann and J.-S. Caux, Phys. Rev. A 89, 033601 (2014).

8. G. Goldstein and N. Andrei, arXiv:1405.6365.

9. G. Goldstein and N. Andrei, arXiv:1309.3471.

10. T.-L. Ho, Phys. Rev. Lett. 81, 742 (1998).

11. T. Ohmi and K. Machida, J. Phys. Soc. Jpn. 67, 1822 (1998).

12. G. Delfino, J. Phys. A 37, R45 (2004).

13. B. Pozsgay, arXiv:1406.4613.

14. B. Wouters et al., arXiv:1405.0172.

15. M. Brockmann et al., arXiv:1408.5075.

16. B. Pozsgay et al., arXiv:1405.2843.

17. G. Goldstein and N. Andrei, arXiv:1405.4224. 